\title{
The Variability of Optically Selected QSO Sample
}

\author{
Keliang Huang and Hongnan Zhou \\ Physics Department, Nanjing Normal University, Nanjing 210097, PRC
}

\section{Introduction}

Variability may be a common property of AGNs. It provides a powerful tool to study the physics of AGNs. For QSOs, variability is a suitable criterion for selecting candidates. Many variability studies on quasar samples have been made (e.g. Netzer and Sheffer 1983, User et al. 1983, Pica and Smith 1983, Wampler and Ponz 1985, Neugebauer et al. 1989, Huang et al 1990, Cristiani et al. 1990, Giallongo et al. 1991, Cimatti et al. 1994, Treverse et al. 1994, Hook et al. 1994, Cristiani et al. 1996, Axetxage et al. 1997). In some studies, a large percentage of quasars is found to have detectable variation. However, other studies find no variability evidence. Many studies also discuss the correlations of variability with luminosity or redshift. Some interesting results have been made. However, up to now, there are no positive conclusions. Further study on variability of quasar samples is obviously needed. In this paper, we report on a variability study of 32 members at the Medium Bright Quasar Sample (Mitchell et al. 1984). The study is actually a continuation of the previous study (Huang et al. 1990).

\section{Observational Data}

The previous study (Huang et al. 1990) is conducted with the help of an uniform series of blue-sensitive filtered plates taken with the Palomar Schmidt telescope spanning the years 1978-1981. The variability is characterized by a canonical standard deviation for each time series and an extremum statistic Q.

Since 1993, we made CCD photometry on the 32 members of the MBQS, using the $1 \mathrm{~m}$ telescope of Yunnan Observatory and the $1.56 \mathrm{~m}$ telescope of Shanghai Observatory. B mag. of the 32 quasars is obtained by use of standard procedure.

\section{The Results and Discussion}

We obtained the B mag. of 32 quasars in each year over 1993-1996. Q-statistic is still used to characterize the variability. Combining the previous results over 1978-1981, the $\mathrm{Q}$-values and maximum amplitude of variation $\left(\Delta B_{\max }\right)$ can be obtained.

We find that 12 quasars, i.e. about $40 \%$ of the total quasars, vary over an $18 \mathrm{yr}$. baseline if $Q \geq 5$ is taken as variability criterion. 
Using the data of 12 variable quasars, we can analyse the relationships between variability and luminosity and between variability and redshift. Taking $q_{0}=0, H_{0}=50 \mathrm{~km} \mathrm{sec}^{1} \mathrm{Mpc}^{-1}$, we find that variability is strongly correlated with absolute magnitude. The regression results are:

$$
(\Delta M)_{\max }=0.026 M_{\max }+1.29,(\gamma=0.72)
$$

Here $(\Delta M)_{\max }$ and $M_{\max }$ are the maximum variation amplitude and the maximum absolute magnitude for the 12 quasars. $\gamma$ is the regression coefficient. The regression is significant at $\alpha=0.01$. Also we can use $\mathrm{Q}$ instead of $(\Delta M)_{\max }$ and make regression. The result is:

$$
Q=0.44 M_{\max }+17.80,(\gamma=0.85)
$$

The regression is also significant at $\alpha=0.01$.

Figure 1 shows $\left((\Delta M)_{\max }, M_{\max }\right)$ and $\left(Q, M_{\max }\right)$ for the variable quasars.

The results above show that there is a strong anticorrelation between variability and luminosity, in the sense that more luminous quasars show less variability. It confirms previous results.

Figure 2 shows $\left((\Delta M)_{\max }, Z\right)$ and $(Q, Z)$ for all variable quasars. Similarly, we make regression analysis. We find:

$$
\begin{gathered}
(\Delta M)_{\max }=-0.092 Z+0.69,(\gamma=0.67) \\
Q=-1.71 Z+7.71,(\gamma=0.85)
\end{gathered}
$$

Both regressions are significant at $\alpha=0.05$.

The results above seem to show there is an anticorrelation between variability and redshift, in the sense that more distant quasars show less variability. However, the apparent magnitudes of MBQS have a maximum difference less than $2 \mathrm{mag}$. and their redshifts spread over 0.06-2. Therefore, more distant quasars are generally more luminous for MBQS. The relationship between variability and redshift obtained above reflects partly the relationship between variability and luminosity. Eliminating the effect of magnitude-redshift, we find no significant correlation between variability and redshift. More detailed analysis is in progress.

Acknowledgments. This work is partly supported by National Climbing Program and NSF of China. 


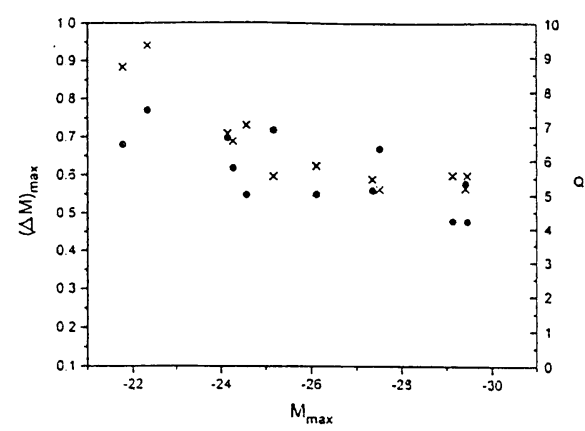

Fig.1 $(\Delta M)_{\max }(\bullet)$.Vs. $M_{\max }$ and $\mathrm{Q}(\times)$.Vs. $M_{\max }$

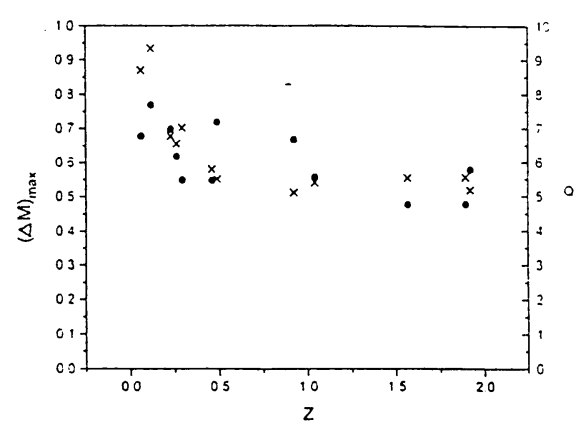

Fig.2 $(\Delta M)_{\max }(\bullet)$.Vs. $Z$ and $Q(x)$.Vs. $Z$

\section{References}

Axetxage et al., 1997, MNRAS, 286, 271

Cimatti, A., Zomorani, G. and Marano, B., 1993, MNRAS, 263, 236

Cristiani, S., Vio, R., and Andreasni, P., 1998, AJ, 100, 56

Cristiani, S., Trentini, S., et al. 1996, A\&A, 306, 395

Giallongo, E., Trevese, D., Vagnetti, F., 1991, ApJ, 337, 345

Hook, I.M., et al., 1994, MNRAS, 268, 305

Huang, K.L., Mitchell, K.J., and User, P.D. 1990, ApJ, 362, 33

Mitchell, K.J. eta 1., 1984, ApJ(letters), 273, L59

Neugebauer, G., et al. 1989, AJ, 97, 957

Netser, H. and Sheffer, T., 1983, MNRAS, 203, 935

Pica, A.J. and Smith, A.G., 1993, ApJ, 272, 11

Trevese, D., Kro, R.G., et al., 1994, ApJ, 433, 494

Usher, P.D. et al., 1983, ApJ, 269, 73

Wamper, E.J and Ponz, D., 1985, ApJ, 298, 448 